\title{
Generation of a Monodisperse Microbubble Jet in Microgravity
}

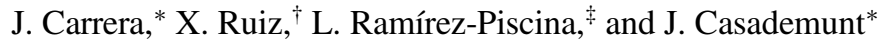 \\ Institut d'Estudis Espacials de Catalunya, \\ 08034 Barcelona, Spain \\ and \\ M. Dreyer $\underline{\S}$ \\ Universität Bremen, 28359 Bremen, Germany
}

\begin{abstract}
DOI: $10.2514 / 1.33283$
A new method to create a jet of a virtually monodisperse microbubble suspension of prescribed bubble size into a quiescent cavity is proposed. The method is insensitive to gravity and is based on the creation of a slug flow at a $\mathrm{T}$ junction in a capillary tube before injection. We develop a theoretical analysis that establishes the validity and efficiency of the method, as controlled by the crossflow Weber number, and yields a simple explicit prediction for the bubble size in terms of the injection parameters. The method operates efficiently for small Weber numbers, yet it generates small bubbles of very uniform size. The reduced size dispersion is also explained within the theoretical model. The method of bubble formation, injection, and spreading by the resulting turbulent jet is validated experimentally in $4.7 \mathrm{~s}$ of free fall in the drop tower at the University of Bremen. Experiments demonstrate the physical principle behind the method of bubble formation and allow us to explore the dynamics of the resulting bubble jet after injection of the slug flow into a quiescent cavity in microgravity conditions as an efficient method of bubble spreading and transport. Detailed measurements of average local velocities show that bubbles are essentially passive with respect to the carrier mean flow, and the inherent turbulence of the flow is crucial for optimal spreading of the bubble distribution and reduction of bubble coalescence. The shape of the bubble jet is studied as a function of the Reynolds number. Finally, the degree of coalescence is also characterized and found to be remarkably small.
\end{abstract}

\section{Nomenclature}

$A$
$d$
$g$
$J$
$Q$
$u$
$\alpha$
$\mu$
$\rho$
$\sigma$

$=$ cross-sectional area

injector diameter

acceleration of gravity

injected momentum rate

volumetric flow rate

velocity in the $x$ direction

auxiliary parameter

dynamic viscosity

density

surface tension

\section{Subscripts}

$$
\begin{array}{lll}
B & = & \text { bubble } \\
g & = & \text { gas phase } \\
l & = & \text { liquid phase }
\end{array}
$$

\section{Introduction}

D URING the last decades, the power demands of spacecraft have increased dramatically. The future of different space endeavors involving manned missions will require more efficient heat and mass transport technologies to significantly improve space system designs. The replacement of single-phase flow with two-phase flow systems could be a feasible option to increase these efficiencies, if significant stronger efforts are made to bring multiphase-flow-based

Received 11 July 2007; revision received 17 February 2008; accepted for publication 10 April 2008. Copyright $\odot 2008$ by the American Institute of Aeronautics and Astronautics, Inc. All rights reserved. Copies of this paper may be made for personal or internal use, on condition that the copier pay the $\$ 10.00$ per-copy fee to the Copyright Clearance Center, Inc., 222 Rosewood Drive, Danvers, MA 01923; include the code 0001-1452/08 \$10.00 in correspondence with the CCC.

*Universitat de Barcelona, Barcelona, Spain.

Universtitat Rovira i Virgili, Tarragona, Spain.

*Universitat Politècnica de Catalunya, Barcelona, Spain.

${ }^{\S}$ Center of Applied Space Technology and Microgravity. systems to the desired Technology Readiness Levels. More efficient and predictable multiphase-flow-managing techniques will enhance their space utilization in 1) environmental control to improve efficiency and reliability, 2) life support systems to support technology of human exploration and development of space, 3 ) bioreactors to obtain a better homogeneous oxygenation of cellgrowing media, and 4) chemical contactors or propulsion systems, to maximize the contact-area-to-volume ratio.

On Earth, submerged gas injection has been extensively studied for many years, becoming a well-understood and ubiquitously used industrial process. To find an exhaustive source of literature in bubble generation under normal gravity, refer to the reviews by Rabiger and Vogelpohl [1] and Kumar and Kuloor [2]. On the contrary, gas injection in microgravity is still poorly understood, partly due to significant gaps of experimental data. Also integral for managing two-phase flows in microgravity environments are the techniques and systems used for bubble generation. In space, due to the absence of buoyancy, auxiliary systems must be used to help bubble detachment from the injector, and thus in the early 1990s, the first efforts to gather empirical evidence to elucidate the process of bubble generation in microgravity were carried out on parabolic flights and drop towers. Those studies revealed that simple submerged gas injection (employing the systems used on Earth) was not technologically feasible for space systems, due primarily to the resulting bubbles being excessively large and prone to coalescence. As a result, systems employing liquid coflow or crossflow configurations, or even more elaborate methods such as electric and acoustic fields, have been studied to aid bubble detachment under microgravity conditions.

In particular, Kim et al. [3] were among the first to study the bubble-formation mechanism in microgravity through theoretical two-stage models in flowing liquids. Their predictions agreed satisfactorily with most of the available experimental data under normal gravity and proposed that bubble detachment in the absence of buoyancy could only be achieved by the drag force generated by a liquid flow. This assumption was rapidly abandoned in favor of the experimental results of Pamperin and Rath [4]], studying the influence of buoyancy on bubble formation from submerged orifices in liquids. Their investigation compared the results of bubble 
formation under normal and microgravity, revealing that bubble detachment in quiescent liquids under microgravity was possible at high gas flow rates, corresponding to $W e_{g}>8$ (later recalculated [5] to $\left.W e_{g}>4\right)$, where $W e_{g}=\left(\rho_{g} u^{2} d_{g}\right) / \sigma$ is the Weber number associated with the gas flow. Their theoretical interpretation of this result was crucial to demonstrate that bubble detachment was possible in the absence of buoyancy, even though the experimental outcome was not fully satisfactory for practical applications. A nearly parallel study by Buyevich and Webbon [6] investigated bubble formation from submerged orifices to evaluate the effects of fluctuating gravity levels, as well as the influence of other factors, when buoyancy was partially or totally removed. The effects of surface tension, gas pulsation, and injector geometry on bubble formation were also examined, revealing that the force contributions during bubble detachment caused by the surface tension cancel each other. Likewise, Tsuge et al. [7] found that surface tension tended to keep bubbles spherical, but did not affect the growth rate. In those experiments, performed at small gas flows $\left(W e_{g} \leq 0.071\right)$ in quiescent liquid, bubble detachment was not observed. In additional experiments with larger gas fluxes [8], they studied the effects of different liquid flow conditions in the growth and detachment of bubbles. Bhunia et al. [9] concluded that gas momentum enhanced bubble detachment, whereas surface tension inhibited bubble detachment. In contrast, the liquid drag and the inertial forces acted as enhancing or inhibiting factors for bubble detachment, depending on the relative velocities of the bubbles and the flowing liquid. The effects of coflow and crossflow configuration were revisited by Pais et al. [10], showing that independently of the flow configuration, bubble size decreased with increasing liquid superficial velocity, whereas bubble size increased with injector nozzle diameter and gas flow rates. A similar study was conducted by Nahra and Kamotami [11] on the effects of liquid crossflow, gas flow rate, and orifice diameter, reaffirming the most significant findings of Pais et al. [10]. Carrera et al. $[12,13]$ found that bubble detachment in microgravity environments was possible even at low gas flow rates $\left(W e_{g}=0.2\right)$. This difference with respect to the results of Pamperin and Rath [4] was attributed to geometric considerations that lead to significant variations in the bubble-detachment mechanics. Finally, the effect of electric fields on bubble detachment was also studied by Herman et al. [14,15] and Di Marco et al. [16]. For example, the latter investigation showed that the application of electric fields $(0-18 \mathrm{kV})$ was effective in promoting bubble detachment, showing that electric fields of $10 \mathrm{kV}$ generated bubbles of the same size as in normal gravity. However, as the bubbles left the core of the electric field, bubble coalescence was unavoidable.

In the present work, we study the mechanism of bubble detachment in the presence of a liquid crossflow and the subsequent spreading of bubbles in a quiescent liquid under microgravity, aiming at new possibilities for the future utilization of two-phase flow systems in space hardware. Our main objective is to devise a multipurpose method for formation of bubbles of small sizes (on the order of $1 \mathrm{~mm}$ or smaller) that is insensitive to gravity and that allows an efficient control of the bubble size. Ideally, the bubble should arrange in a suspension of monodisperse bubbles in a jet in which bubble coalescence would not be statistically significant and that could then be conducted for different uses.

The key idea is to use a crossflow arrangement with capillary tubes in a $\mathrm{T}$ junction. The liquid crossflow would then be responsible for bubble detachment, but for sufficiently large surface tension, the formed bubbles would be of a size close to the tube diameter: that is, a slug flow. As a result, what is to be injected in the quiescent cavity, rather than just the gas, is a gas-liquid nearly periodic sequence with a small injection rate, thus creating a bubbly conic jet with a relatively small Reynolds number. For practical purposes, it will be interesting to generate bubbles as small as possible and as monodisperse as possible.

Our method adapts the concept of liquid crossflow reported by Bhunia et al. [9], Pais et al. [10], and Nahra and Kamotani [ $\underline{5}, 11]$ by further exploring a new range of conditions in the capillarydominated regime. The competition of gravity and surface-tension forces is expressed through the Bond number

$$
B o=\frac{\Delta \rho g L^{2}}{\sigma}
$$

and in cases in which $B o \ll 1$, the gravity effects become negligible compared with those of surface tension. Thus, to operate in a capillary-dominated regime on Earth as well as in microgravity, a system should have a small length scale ( $L \sim 1 \mathrm{~mm}$ for water). Furthermore, it is crucial to correlate the effects of inertia and drag to surface-tension forces on the bubbles, and this is expressed by the appropriate Weber number calculated in each situation with the relevant parameters. In our setup, it will be relevant to use the crossflow Weber number, defined by the density and velocity of the liquid crossflow, because the drag force that will be responsible for bubble detachment is related to that flow, whereas other forces (e.g., inertial forces of the gas) will be neglected.

In this paper, we also study the bubble jet generated by our method when the slug flow is injected into a quiescent cavity. For this purpose, we performed a series of drop-tower microgravity experiments. In particular, we characterize the bubble spreading and degree of coalescence in a certain range of injection parameters.

Finally, let us remark that the results presented here can also be of interest in a more general context of bubble dynamics from a fundamental point of view and also to more technological applications such as drag-reduction techniques or cavitation problems.

\section{Theoretical Approach to Bubble Formation}

In this section, we present the theoretical framework in which the performance of the proposed method can be understood quantitatively and extrapolated in parameter space. With the help of the experimental data reported in the following sections, we will provide an explicit prediction of bubble diameter as a function of the relevant parameters and an estimation of the reduction of bubble-size dispersion achieved.

Let us consider a $\mathrm{T}$ junction in which the gas is injected (in the $y$ direction) by a nozzle of diameter $d_{g}$ into a liquid crossflow conduct (along the $x$ direction) of diameter $d_{l}$. The nominal Weber number associated with the crossflow will be

$$
W e^{0}=\frac{\rho_{l} u_{l}^{2} d_{l}}{\sigma}
$$

where $u_{l}=Q_{l} / A$ is the mean liquid velocity, and where $Q_{l}$ is the volumetric flow rate of liquid and $A=\frac{\pi}{4} d_{l}^{2}$ is the cross section of the liquid conduct. In the presence of a growing bubble of size $d_{B}$, the liquid flow is constrained to a smaller cross section $A-A_{B}$, where the bubble cross section $A_{B}$ is taken as $A_{B}=\frac{\pi}{4}\left(\alpha d_{B}\right)^{2}$, and where $\alpha$ is a geometric factor of order 1 that, in principle, could depend on the bubble size and on the flux conditions, but that will be taken here as constant. The local liquid velocity will be accordingly increased by flux conservation as $u_{l}^{*}=u_{l} /\left(1-A_{B} / A\right)$. The effective Weber number associated with this modified crossflow and with the scale of the bubble is

$$
W e^{*}=\frac{\rho_{l} u_{l}^{* 2} d_{B}}{\sigma}
$$

Note that any reasonable prescription for the bubble linear size $d_{B}$ is, in principle, valid for this theory, because any multiplicative factor can be absorbed in the value of $\alpha$. In practice, we will use the diameter of a sphere of the same volume $V_{B}=\pi d_{B}^{3} / 6$, because this diameter can be directly measured afterward if the generated slug flow is injected into a cavity.

In general, the detachment process is controlled by the balance of several forces: surface tension, drag, liquid and gas inertia, and lift effects induced by liquid shear. It has been shown [11] that in the regime for which buoyancy and gas inertia can be neglected, detachment is dominated by the balance of forces in the $x$ direction: namely, capillary, drag, and liquid inertia forces. In the regime of interest here, with small flow rates of gas $Q_{g}$ and liquid $Q_{l}$ (and hence small $u_{l}$ ), but a large local velocity $u_{l}^{*}$, liquid drag is strongly 
enhanced and is the dominant detaching force, whereas liquid inertia (depending on $u_{l}$ and on $Q_{g}$ ) can be neglected. In this situation, the analysis simplifies to a simple balance between the $x$ component of the capillary force:

$$
F_{\sigma}=\pi \sigma d_{g} f
$$

where $f$ is a geometric factor and the drag force in the crossflow direction, which takes the form

$$
F_{D}=C \rho_{l}\left(u_{l}^{*}\right)^{2} d_{B}^{2}\left(1+\mathcal{O}\left(\frac{1}{\sqrt{R e_{B}}}\right)\right)
$$

where the constant $C$ could be determined following Moore [17] (see also Magnaudet and Eames [18]). The precise knowledge of it depends on geometric factors and is not required here. Moreover, we will assume the invariance of the bubble shape in the regime dominated by capillarity, and the geometric factors $C$ and $f$ will also be taken as constant. Notice, however, that we assume sufficiently large values of the associated bubble Reynolds number:

$$
\operatorname{Re}_{B}=\frac{\rho_{l} u_{l}^{*} d_{B}}{\mu_{l}}
$$

The basic assumption of the following analysis is that bubble detachment occurs when the ratio of drag to capillary forces $F_{D} / F_{\sigma}$ is of the order of a critical value yet to be determined, within a certain range, also unknown. By scaling all distances with the crossflow diameter $d_{l}$, that is,

$$
\begin{gathered}
\eta=\frac{d_{g}}{d_{l}} \\
\xi=\frac{d_{B}}{d_{l}}
\end{gathered}
$$

this ratio can be written as

$$
\frac{F_{D}}{F_{\sigma}}=\frac{C}{\pi f} W e^{*} \frac{\xi}{\eta}
$$

We then assume that a bubble of linear size $d_{B}$ in the presence of a crossflow of local velocity $u_{l}^{*}$ will detach whenever

$$
W e^{*} \frac{\xi}{\eta}=W e^{c}
$$

where $W e^{c}$ is a critical Weber number value, with an uncertainty $\delta W e^{c}$. Note that $\xi / \eta$ is the ratio of bubble to gas nozzle diameters, and in the desired operating mode (small bubbles), this ratio should be of order 1. Taking into account the effect of the bubble cross section, the effective crossflow Weber number can be written as

$$
W e^{*}=W e^{0} \frac{\xi}{\left(1-(\alpha \xi)^{2}\right)^{2}}
$$

We see in this equation that for small $W e^{0}$, the detachment condition can be fulfilled only if the denominator in its right-hand side is also small, which corresponds to the bubble being close to filling the whole section of the tube. In this situation, even for small fluxes, the local velocity $u_{l}^{*}$ can achieve arbitrarily large values, and $W e^{*}$ can exceed the critical value $W e^{c}$ for detachment. Bubble size is then controlled by the tube diameter.

By defining the nondimensional parameter

$$
\epsilon=\left(\frac{W e^{0}}{\eta W e^{c}}\right)^{1 / 2}
$$

the detaching condition can then be written as

$$
\epsilon \xi=1-(\alpha \xi)^{2}
$$

Resolution of Eq. (13) will provide the relative size of the detached bubbles for a given value of $\epsilon$. This parameter, which is proportional to $u_{l}$, plays the role of a nondimensional crossflow velocity and will define the operating regime of the system. Equations (12) and (13) are the basic equations to understand the performance of our method. They relate the size of the detached bubbles with the nominal Weber number and have two unknown parameters, $\alpha$ and $W e^{c}$.

In our proposed setup, both gas and liquid conducts are of similar capillary dimensions; that is, $\eta$ is of order 1 , and volumetric flow rates of both gas and liquid are also of similar order. We are interested in the limit of small injection fluxes. That corresponds to a small Weber number, $W e^{0} \ll W e^{c}$ or, in Eq. (13), small $\epsilon$. In that limit, the solution of Eq. (13) is $\xi=1 / \alpha$. That means $d_{B}=d_{l} / \alpha$ or also $A_{B}=A$; that is, the bubble detaches when it occupies all the available liquid cross section. As already explained, the rapid increase of the effective cross velocity $u^{*}$ and the associated Weber number $W e^{*}$ when $A_{B}$ approaches $A$ is ultimately the cause of the detachment of the bubble. We will see subsequently that this also ensures that the intrinsic uncertainty $\delta W e^{c}$ corresponds to a much narrower uncertainty $\delta \xi$ for bubble size.

The dependence of the bubble size on the crossflow can be obtained from the solution of Eq. (13):

$$
\xi=\frac{1}{\alpha}\left(1+\frac{\epsilon^{2}}{4 \alpha^{2}}\right)^{1 / 2}-\frac{\epsilon}{2 \alpha^{2}}
$$

Operating at small fluxes, we can take the first order in $\epsilon$ of Eq. (14), which, in terms of the Weber number, can be written as

$$
\xi=\frac{d_{B}}{d_{l}}=\frac{1}{\alpha}-\frac{1}{2 \alpha^{2}}\left(\frac{W e^{0}}{\eta W e^{c}}\right)^{1 / 2}+\mathcal{O}\left(\epsilon^{2}\right)
$$

The dimensionless bubble size is thus of order 1 , with a weak decreasing dependence on $\left(W e^{0} / W e^{c}\right)^{1 / 2}$. Equation $(\underline{15})$ is the main prediction of our theoretical analysis. It contains two unknown parameters that will be determined from experimental fits.

The second prediction of our analysis concerns the dependence of the bubble-size dispersion. In fact, the regularity of the bubblegeneration process can be assessed by working out the relative dispersion of bubble sizes. We have already introduced the range $\delta W e^{c}$ of values of critical Weber number at which bubbles actually detach. Differentiating Eq. (15), we obtain, to leading order in $\epsilon$,

$$
\frac{\delta d_{B}}{d_{B}}=\frac{\delta \xi}{\xi}=\frac{\epsilon}{4 \alpha} \frac{\delta W e^{c}}{W e^{c}}+\mathcal{O}\left(\epsilon^{2}\right)
$$

A crucial feature of this result is the presence of the $\epsilon$ factor in its right-hand side. We see that dispersion of bubble sizes can be arbitrarily reduced by operating in the small flux regime $\epsilon \ll 1$ or, equivalently, $W e^{0} \ll W e^{c}$.

To illustrate the distinctive performance of this particular setup with respect to previous approaches, we will apply Eq. (13) to the standard crossflow setup, in which a small nozzle injects gas into a liquid tube of much larger diameter with the aim of generating small bubbles. In the latter case, and depending on flow parameters, the neglected effects of liquid and gas inertia could be important, and therefore the present analysis is only estimative. We also ignore the reduction in liquid velocity occurring near the tube walls, which could be significative in the detaching of small bubbles. For this case, $d_{g} \ll d_{l}(\eta \ll 1)$. The regime of small bubbles, which ideally should be of sizes comparable with that of the gas nozzle, corresponds then to small $\xi$, because $\xi \sim \eta \ll 1$. To achieve such small bubbles, Eq. (13) indicates that a large flux is necessary: $\epsilon \sim 1 / \xi \gg 1$ or $W e^{0} \gg W e^{c}$. Because of the reduction of velocity on the walls, actual fluxes should be even much larger. Therefore, the standard crossflow operation indeed corresponds to a limit that is opposite to ours. In the limit of large $\epsilon$, Eq. (14) reduces to $\xi=1 / \epsilon+\mathcal{O}\left(\epsilon^{0}\right)$, which, after differentiation for the relative dispersion of bubble sizes, gives

$$
\frac{\delta d_{B}}{d_{B}}=\frac{\delta \xi}{\xi}=\frac{1}{2} \frac{\delta W e^{c}}{W e^{c}}
$$



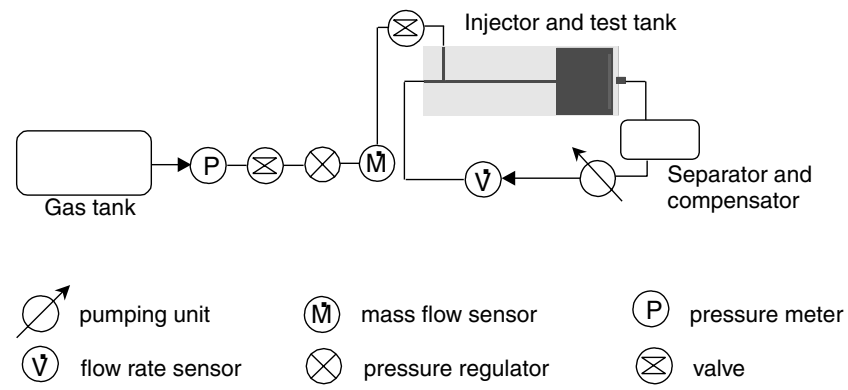

Fig. 1 Sketch of the experimental setup.

For small $W e^{0}(\epsilon \ll 1)$, with our setup, we thus obtained a significant noise-reduction prefactor (of order $\epsilon$ ) compared with the standard regime. In addition, note that for fixed surface tension, a larger Weber number also implies a larger Reynolds number, and so the inherent noisiness of the detachment events (captured in this framework by $\delta W e^{c}$, but closely related to the degree of turbulence of the crossflow) is expected to be even larger for large $W e^{0}$.

We conclude this section by providing the values of the unknown parameters that we fitted from the experimental data presented in the following sections. We obtain a critical Weber number of $W e^{c}=$ $36 \pm 8$ and a geometrical factor $\alpha=0.68 \pm 0.03$. The noisereduction factor $\epsilon /(2 \alpha)$ ranges in our experiments from 0.07 in the most favorable case to 0.37 in the less favorable one.

\section{Experimental Setup}

Experimental information was collected in the 4.74-s University of Bremen drop tower, offering a microgravity quality better than $10^{-5} \mathrm{~g}$. To carry out the experiments, the setup was integrated into a drop-capsule structure that was kept under atmospheric pressure and temperature. Reference ground experiments under $1 g$ were also conducted for comparing the results in the two environments.

The experimental setup sketched in Fig. 1 consisted of a squared Plexiglas test tank, with a base of $100 \times 100 \mathrm{~mm}^{2}$ and a height of $100 \mathrm{~mm}$, containing a volume of 11 . The gas and liquid T section in which bubble detachment occurred was $1.5 \mathrm{~mm}$ in diameter. The gas-liquid composite channel from the T section to the test tank had also a diameter of $1.5 \mathrm{~mm}$ and a length of $205 \mathrm{~mm}$ and was attached at the geometric center of the test tank base. The chosen length of $205 \mathrm{~mm}$ (greater than 130 injector diameters) is sufficiently large to ensure stationary slug-flow conditions.

Dry filtered air and deionized water were used in all experiments. No treatment such as deaerating or extensive bubbling was applied, taking into account that no significant changes of pressure and temperature were expected and that the experiment times were too short for significant mass transfer to occur. The gas phase was stored in a pressure tank. Control and measurement of the desired gas throughput for each experiment was achieved by an electromechanical pressure regulator, together with a precision orifice. With this arrangement, and maintaining sonic velocity at the orifice inlet, the gas mass flow rate is a direct function of the orifice stagnation pressure. In addition, this configuration eliminates the effects derived from downstream pressure changes upon the desired volumetric gas flow rates $[1,2]$. The liquid phase was pumped by a magnetically driven gear pump with adjustable speed for selecting the desired liquid flow rates, which were measured by an electronic volume sensor. A volume-compensation vessel was installed downstream of the liquid test tank, to compensate for the additional volume of gas injected into the system throughout the experiments. This unit maintained constant pressure in the system, regardless of the gas/ liquid injection rates, and also prevented the formation of free surfaces while in microgravity. Additionally, to mitigate sudden pressure changes in the system induced by the rapid transition from $1 \mathrm{~g}$ to microgravity, a liquid closed-loop layout was preferred, in which the test section and composite injector length were placed horizontally. The horizontal scheme of all liquid lines and components minimized the negative effects during the onset of microgravity. To operate in a liquid closed-loop mode, the volumecompensation vessel also functioned as a gas-liquid separator by venting any excess gas and allowing the liquid phase to recirculate.

The experimental program, shown in Table 1 , includes variations of gas and liquid volumetric flow rates, by selecting an envelope of conditions that are characteristic of generating slug-flow patterns in microchannels. We also include the momentum injection rate $J$ as the main parameter characterizing the jet structure in the cavity in the absence of bubbles [19]. Neglecting the momentum carried by the gas, the momentum injection rate is given by $J=\rho_{l} Q_{l}\left(Q_{l}+Q_{g}\right) / A$. Note that although only the liquid carries momentum, the injection of gas contributes to the momentum because it accelerates the liquid. In the preceding expression of $J$, it is also assumed that the gas is incompressible. For the computation of the nominal Weber number $W e^{0}$, we used the value of surface tension at $20^{\circ} \mathrm{C}$ : $\sigma=72.8$ dyne $/ \mathrm{cm}$. The Reynolds number is also an important number in our experiment because it controls the bubble spreading. Although the Reynolds number associated with the liquid in the capillary tube, which is relevant to the nature of the pipe flow, should read $R e_{l}=\left(Q_{l}+Q_{g}\right) d /(A v)$, with $v=10^{-2} \mathrm{~cm}^{2} / \mathrm{s}$, the relevant definition of the velocity scale in the jet is given by the injected momentum as $\sqrt{J /(\rho A)}$. Because the volume fraction of gas is much smaller in the region occupied by the jet than in the capillary, we can define the relevant Reynolds number for the jet as

$$
R e=\frac{2 \sqrt{J /(\pi \rho)}}{\nu}=\frac{4 \sqrt{Q_{l}\left(Q_{l}+Q_{g}\right)}}{\pi \nu d}
$$

Because the transversal size of the jet grows linearly with distance $x$, whereas the velocity decreases as $1 / x$, the effective Reynolds number is roughly the same in the whole jet. In our analysis, we also disregard the amount of liquid that may be vaporized into the bubbles as a negligible effect within the overall uncertainty of the experiment (which comes mainly from the fluctuations of the flow rates). Two digital high-speed video cameras (500 frames per second) recorded the bubble-formation process in microgravity: one camera for a detailed view of the gas-liquid composite injector before entering the test tank and a second one for a global view of the test tank. Illumination of the two areas of interest was achieved by a backlight and a light-diffuser arrangement. Analysis of the results was done by

Table 1 Experimental parameters used in the drop-tower experiments-

\begin{tabular}{lccccccccc}
\hline \hline Test & $Q_{l}, \mathrm{~cm}^{3} / \mathrm{s}$ & $Q_{g}, \mathrm{~cm}^{3} / \mathrm{s}$ & $u_{l}, \mathrm{~cm} / \mathrm{s}$ & $u_{g}, \mathrm{~cm} / \mathrm{s}$ & $J, \mathrm{gcm} / \mathrm{s}^{2}$ & $W e^{0}$ & $R e$ & $d_{B}, \mathrm{~cm}$ & $d_{B}^{\prime}, \mathrm{cm}$ \\
\hline 1,2 & 0.69 & 0.27 & 39.0 & 15.3 & 37.48 & 3.14 & 815 & 0.180 & 0.204 \\
3 & 1.23 & 0.30 & 69.6 & 17.0 & 106.49 & 9.98 & 1164 & 0.138 & 0.147 \\
4 & 0.45 & 0.26 & 25.5 & 14.7 & 18.08 & 1.34 & 480 & 0.197 & 0.214 \\
5 & 0.91 & 0.32 & 51.5 & 18.1 & 63.34 & 5.46 & 898 & 0.166 & 0.199 \\
6 & 0.58 & 0.16 & 32.8 & 9.1 & 24.29 & 2.22 & 556 & 0.181 & 0.190 \\
7 & 0.26 & 0.15 & 14.7 & 8.5 & 6.03 & 0.45 & 277 & 0.210 & 0.225 \\
8 & 0.51 & 0.16 & 28.9 & 9.1 & 19.34 & 1.72 & 496 & 0.190 & 0.228 \\
9 & 0.85 & 0.25 & 48.1 & 14.1 & 52.91 & 4.77 & 821 & 0.170 & 0.213 \\
10 & 0.52 & 0.21 & 29.4 & 11.9 & 21.48 & 1.78 & 523 & 0.187 & 0.240 \\
11 & 0.54 & 0.21 & 30.6 & 11.9 & 22.92 & 1.92 & 540 & 0.183 & 0.205 \\
\hline \hline
\end{tabular}

a See the definitions in the text; average bubble diameters are measured right after detachment $d_{B}$ and after $4 \mathrm{~s}$ of microgravity $d_{B}^{\prime}$. 
digital image processing software. Finally, note that the injection procedure of gas and liquid was started $10 \mathrm{~s}$ before the onset of microgravity to stabilize the flow rates. In this way, bubble formation and spreading in microgravity using the proposed injector was investigated for $0.26 \leq Q_{l} \leq 1.23 \mathrm{~cm}^{3} / \mathrm{s}$ and $0.15 \leq Q_{g} \leq$ $0.32 \mathrm{~cm}^{3} / \mathrm{s}$, corresponding to $14.7 \leq u_{l} \leq 69.6 \mathrm{~cm} / \mathrm{s}$ and $8.5 \leq u_{g} \leq 18.1 \mathrm{~cm} / \mathrm{s}$. Under all the conditions tested, detachment from the injector was naturally achieved, and the generated bubbles were actively spread in the interior of the liquid test tank. Tests 1 and 2 were performed with the same flow conditions to assure that the experimental apparatus could produce repeatable and accurate data in weightlessness. The measured data for $d_{B}$ and $d_{B}^{\prime}$ correspond to the average of the two experiments.

\section{Results on Bubble Formation}

In Fig. 2 we show a sequence of snapshots of the bubble spreading in the liquid test tank under microgravity for a representative case (test 2). The bubbles formed under microgravity were nearly spherical, with a diameter roughly equal to the diameter of the injector, and only weak deformations were occasionally observed when the bubble relative velocity with the fluid was sufficiently high to deform them by shear stress. Bubbles generated in the seconds of normal gravity before drop release appear in an ascending column in the first snapshots and accumulated at the top of the cavity. Bubbles traveling away from the injector in microgravity generate a conicalshape distribution, with the apex positioned at the injector. This kind of distribution is maintained for large distances downstream, with no significant clustering. Bubbles generated and spread under normal gravity have nearly the same size and are roughly equal to the injector diameter, even though the bubble jet shape is obviously radically different. In Fig. 3 we show snapshots of bubble distributions for different drop tests at equivalent times to provide a visual grasp of the typical bubble jets obtained and an overall impression of their quality, variability, and noisiness.

Bubbles larger than those of nominal size are relatively few and are usually due to coalescence events, but in some cases, they can be traced back to sporadic dysfunction of the injection mechanism, mostly of the gas pump, originating anomalous fluctuations of the injection flow rates.

Figure 4 shows three snapshots of the slug flow at the end of the capillary injector and the behavior of the bubbles at the entrance of
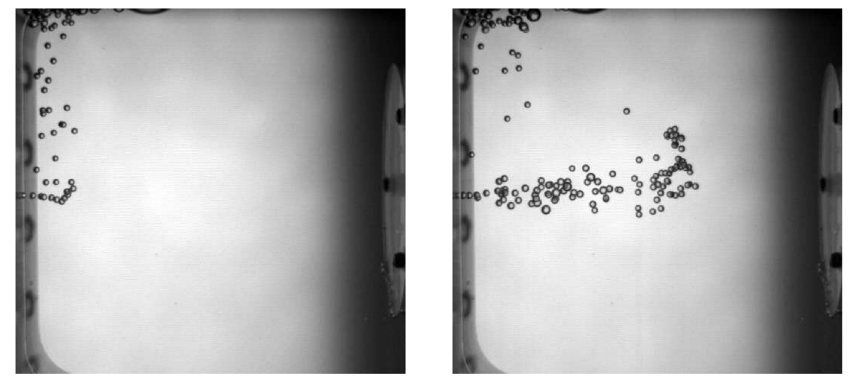

a)

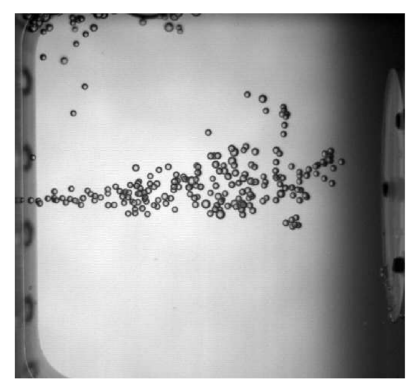

b)

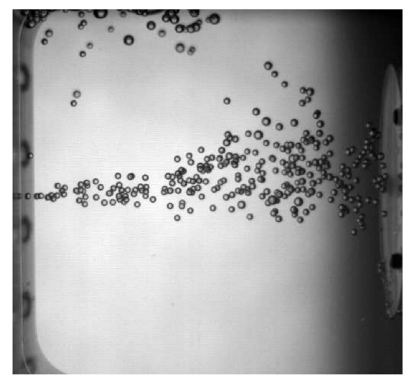

d)

c)

Fig. 2 Snapshots of test 2: a) $t=0.044 \mathrm{~s}$, b) $t=0.772 \mathrm{~s}, \mathrm{c}) t=1.500 \mathrm{~s}$, and d) $t=2.228 \mathrm{~s}$ (times in seconds after capsule release, after $10 \mathrm{~s}$ of injection in normal gravity).

the quiescent cavity. The bubble trains are quite periodic and stationary. A slight change of bubble volume can be observed, corresponding to the different values of injection parameters.

Determination of bubble sizes from video images (see Table 1) shows excellent agreement between the theoretical prediction Eq. (15) and the experiments, both in normal gravity and microgravity, as shown in Fig. 5. The dimensionless bubble size is indeed close to 1 and exhibits a linear decrease on $\left[W e^{0}\right]^{1 / 2}$. Results in the two gravity environments do not show significant differences. From these data, we can obtain a fit of the unknown parameters of the theory: namely, the geometrical factor $\alpha=0.68 \pm 0.03$ and the critical Weber number $W e^{c}=36 \pm 8$.

A quantitative characterization of the slug flow generated at the capillary T junction is shown in Figs. $\underline{6}$ and $\underline{7}$. Figure $\underline{6}$ shows the dependence on the liquid velocity of the $\bar{t}$ wo lengths that characterize the unit cell of the slug flow: namely, the longitudinal bubble size and the separation between bubbles. Consistently with our theoretical framework, the analysis exhibits a rather weak dependence of the bubble size on $W e^{0}$. Consequently, the changes in the injection rates translate into a stronger variation of the bubble separation, due to mass conservation. Although not shown here, the monodispersivity of the bubble size is even more pronounced in the capillary slug flow than in the cavity, because no coalescence events occur in this regime.

Figure 7 shows the frequency of bubble formation as a function of the crossflow liquid velocity. A roughly linear increase from around 25 to almost 200 bubbles per second is observed as the liquid velocity increases from 15 to $70 \mathrm{~cm} / \mathrm{s}$. The fact that such frequency exhibits a linear growth is once more a signature of the weak dependence of bubble size on the injection parameters, implying that the time scale of bubble formation is essentially set out by the time scale of the crossflow.

It is worth remarking that the dimensionless bubble size $d_{B} / d_{g}$ in our experiments is quite small compared with related experiments of gas injection. Nahra and Kamotani [5] used the same injector diameter in rather different flow conditions and obtained much larger bubble sizes. This difference is well understood in the context of the theory proposed in Sec. II. In fact, as discussed there, in the cases in which $d_{l} \gg d_{g}$, bubble size has a strong dependence on flow rate values and it would be necessary to reach very large values of liquid velocity to obtain small bubbles of sizes comparable with $d_{g}$. In cases in which $d_{l} \approx d_{g}$, the size of bubbles results to be limited by the

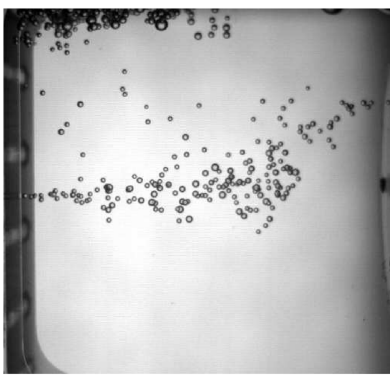

a)

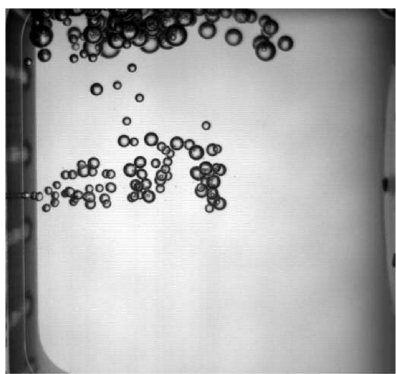

c)

Fig. 3 Snapshots of different drop tests at $\boldsymbol{t}=\mathbf{0 . 7 7 2} \mathrm{s}$ after capsule release: a) test 3 , b) test 4 , c) test 5 , and d) test 7

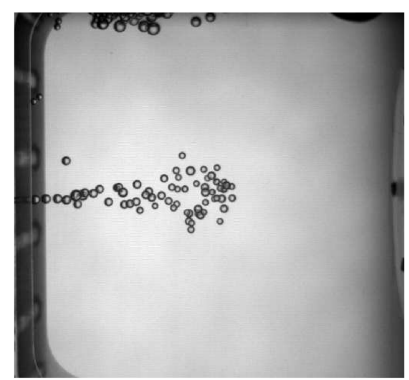

b)

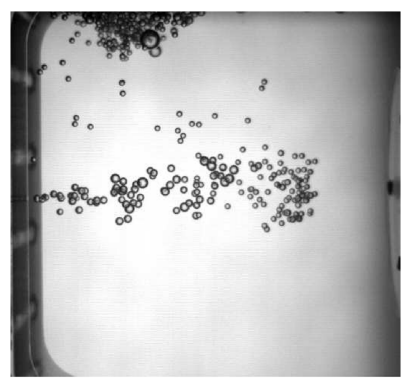

d) 


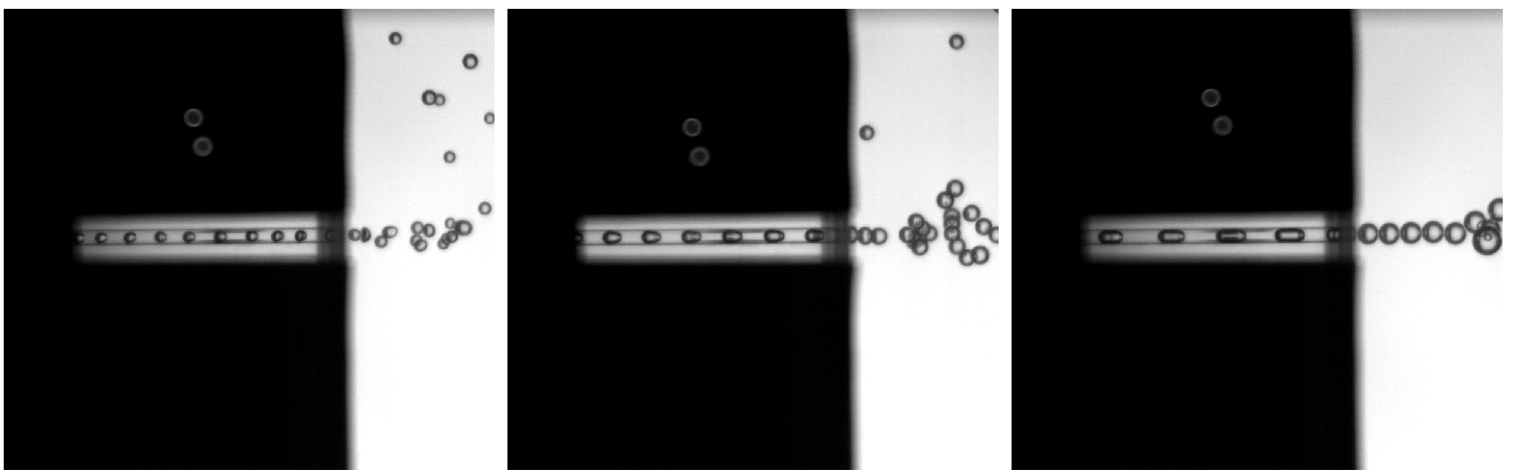

Fig. 4 Representative snapshots of injected slug flow at the inlet during tests 3, 2, and 6.

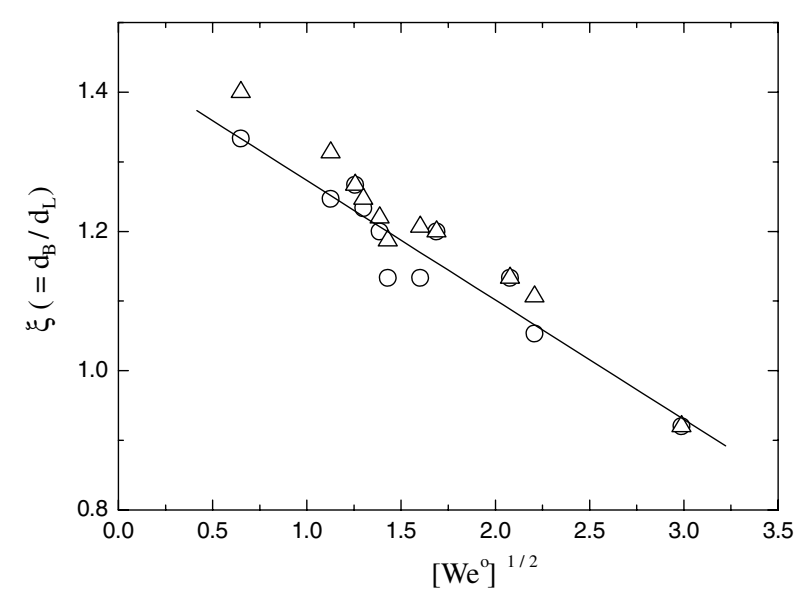

Fig. 5 Dimensionless bubble diameter vs the square root of the Weber number for normal gravity experiments (circles) and microgravity experiments (triangles); fit is based on the theory of Sec. II (straight line); estimated relative error of the slope is $2.5 \%$ and of the intercept is $3.5 \%$.

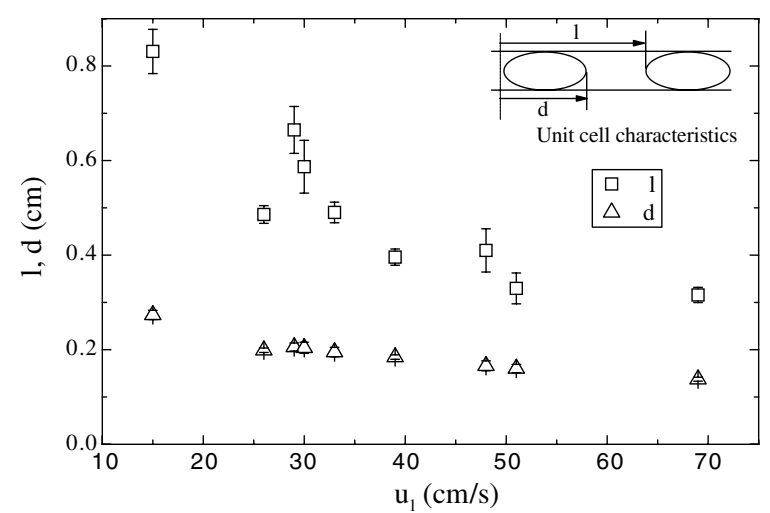

Fig. 6 Quantitative behavior of the two parameters defining the unit cell under conditions of the slug-flow regime.

liquid section for small fluxes. When the bubble is about to fill all of the liquid section, the drag is strongly enhanced, and due to the divergence of the effective crossflow velocity $u^{*}$, the detachment is quickly triggered. In addition, the results reflect that large variations of the crossflow liquid velocity $u_{l}$ only result in small changes of bubble size. Considering that the balance between liquid drag and surface tension is the governing mechanism of bubble detachment, one could expect a much larger difference in bubble size compared

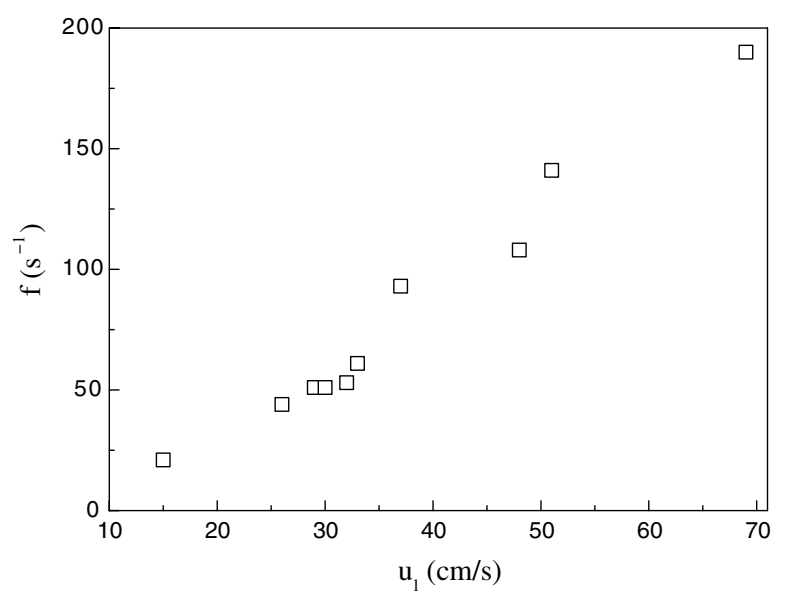

Fig. 7 Bubble frequency formation (in bubbles per second) vs crossflow velocity.

with past investigations. For instance, Pais et al. [10] showed that varying the crossflow liquid velocity from 10 to $60 \mathrm{~cm} / \mathrm{s}$ resulted in a reduction of the bubble diameter $d$ by nearly $300 \%$. On the contrary, in the present experiments, liquid velocity variations from 15 to $69 \mathrm{~cm} / \mathrm{s}$ only reduced bubble diameters by $35 \%$. Once more, it becomes apparent that the mechanism controlling bubble detachment in both scenarios is radically different.

\section{Dynamic Characterization of Bubble Jets}

Once inside the cavity, the injected bubbles are abruptly slowed down, but the inertia carried by the liquid part of the slug flow itself prevents the accumulation of bubbles and creates a turbulent jet that spreads them over a region with an approximately conical envelope. The bubble jet is relatively sparse and bubbles remain spherical (small Weber number). The method proposed for bubble formation thus simultaneously provides a natural and efficient method for bubble spreading and transport.

In all our ranges of variation of Reynolds numbers, the jet formed in the cavity in the absence of bubbles would be indeed turbulent, even though the pipe flow in the capillary injector remains laminar $[20,21]$. As we will see, the turbulence of the jet is crucial to achieve efficient bubble spreading. A common way to describe turbulent nonhomogeneous flows is to decompose the velocity field into a smooth component that describes some type of time-averaged mean flow, varying slowly in space and time, and an erratic component that contains the rapid fluctuations due to turbulence. This idea can be formalized in different ways, allowing for distinct closures of the equations for the turbulent part. One that we will consider subsequently is the so-called $k-\epsilon$ model [20]. This framework will be 
useful to provide a simple rationale to the observations on the bubbly jet. In this context, the motion of bubbles carried by the jet can also be decomposed into a ballistic component given by the mean flow and a diffusive component inherited from the turbulent component. For small bubbles, it is reasonable to assume that bubbles behave essentially as passive tracers of the mean flow, and so the latter is not expected to be modified by the presence of bubbles. For the fluctuating component, given the bubble size and the typical bubble concentrations, they may well modify the statistics of the turbulent fluctuations through hydrodynamic interactions, depending on the bubble size. For sufficiently small bubbles, they could also be expected to behave as passive tracers for the turbulent component, but this might not be the case in our experiments. The quantitative modeling of the bubble diffusivity is clearly a nontrivial issue, which we will not address here. Alternatively, we will show that simple scaling arguments provide useful insights into the physics of the problem and are sufficient to build up a preliminary quantitative understanding of the dynamics of our bubbly jet.

Note that the situation here is radically different from the so-called pseudoturbulence of bubbly flows in normal gravity [22]. There, the strong fluctuations of the flowfield are caused by the inherent instability of the bubble motion due to buoyancy and to the corresponding hydrodynamic interactions. In microgravity bubbly flows such as the ones considered here, however, the situation is quite different. The erratic motion of bubbles is typically more gentle and must be traced back to the intrinsic inertial turbulence of the carrying flow.

For reference in the following discussion, we recall here the Schlichting solution for the mean flow of a stationary turbulent jet [19] in an infinite cavity, injected through a point orifice at $x=0$. The $x$ component of the velocity reads

$$
u=\frac{3}{8 \pi c} \sqrt{\frac{J}{\rho_{l}}} \frac{1}{\left(1+\zeta^{2} / 4\right)^{2}} \frac{1}{x}
$$

where

$$
\zeta=\frac{1}{4 c} \sqrt{\frac{3}{\pi}} \frac{y}{x}
$$

The constant $c$ is determined from experiments and is $c \simeq 0.0161$. Within our physical picture, averaged quantities such as local densities of bubbles or local velocities of bubbles should reflect the mean flow component of the jet. On the contrary, the large-scale structure of the bubble distribution (and, in particular, the statistics of coalescence) will be sensitive to the turbulent component.

We first address the global shape of the bubble jet. The preceding physical picture naturally explains the systematic observation that bubbles spread over a cone with a relatively well-defined angle, and this angle must be increasing with Reynolds number and saturate to a maximum one. In fact, let us first assume, for the sake of discussion, that the motion of bubbles in the direction transversal to the jet axis is essentially diffusive and controlled by a uniform effective diffusion coefficient $D$. Then for a time lapse $T$, the transversal displacement scales as $R \sim \sqrt{D T}$. On the other hand, taking into account that the longitudinal mean velocity scales as $R e / x$ for a jet, the time lapse $T$ scales with the longitudinal displacement $X$ as $T \sim R e^{-1} X^{2}$. Consequently, eliminating $T$, we get $X \sim R$, and therefore the shape of the region occupied by bubbles must be approximately a cone, with $\tan \phi / 2=R / X$. To estimate the angle $\phi$ of the cone, we need to first estimate the effective diffusion coefficient $D$. In the context of a $k-\epsilon$ model, a natural scale for diffusion of a passive scalar takes the form $D \sim v R e$. Therefore, if one assumes a uniform diffusion coefficient, the ratio $R / X=\tan \phi / 2$ becomes independent of the Reynolds number, reflecting the fact that the same velocity scale controls both the transversal (diffusive) and the longitudinal (ballistic) displacements. This simple analysis actually yields an upper bound of the jet angle that depends essentially on the dimensionless coefficient relating $D$ to $v R e$. Values taken from the $k-\epsilon$ model [20] yield estimates of this upper bound around $30 \mathrm{deg}$.

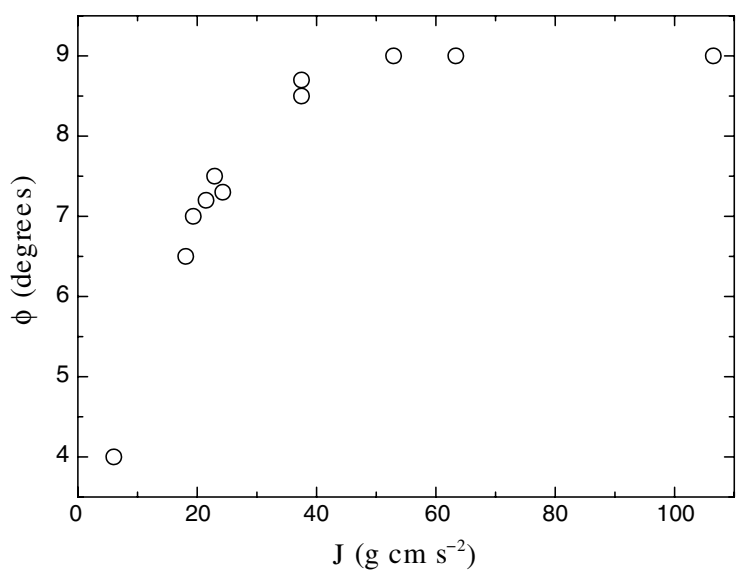

Fig. 8 Jet opening angle vs injected momentum rate $J=\frac{\pi}{4} \rho v^{2} R e^{2}$.

However, note that the turbulent component responsible for bubble spreading is indeed inhomogeneous and, in particular, must vanish at a certain distance from the axis in the region in which the flow is laminar. The angle observed for a bubble jet is thus defining the cone for which turbulent diffusion is significant. Assuming that diffusion is essentially cut off when the flowfield is below a certain velocity scale, the observed angle of the bubble cone is expected to depend on the Reynolds number, which fixes the overall velocity scale of the flow, even though the spatial structure of the flowfield may not depend on it, as captured by the scaling of the mean flow Schlichting solution. The observed dependence of the opening angle with $J=$ $\frac{\pi}{4} \rho v^{2} R e^{2}$ is shown in Fig. $\underline{8}$. Note that it is monotonically increasing with Reynolds number, starting from zero in the laminar case and saturating to a maximum value close to $10 \mathrm{deg}$ at $R e \simeq 800$ $\left(J \simeq 50 \mathrm{gcm} / \mathrm{s}^{2}\right)$. This saturation is a consequence of the fast decay of the flowfield away from the jet axis. The Reynolds number at which the cone angle saturates defines an optimal value for bubble spreading, because it is the minimum Reynolds number that fully unfolds the jet, yet keeping turbulence at a minimum to avoid the potential coalescence as much as possible.

The issue of bubble coalescence is indeed important for practical applications and theoretical interest. It has been pointed out in past investigations in reduced gravity that bubbles had a pronounced tendency to coalesce, forming larger bubbles after detachment $[10,16]$. For practical purposes, this phenomenon is not beneficial because it reduces the effective contact-area-to-volume ratio. In addition, understanding how bubbles can be dispersed in the absence of buoyancy is of high technological interest to successfully design and operate contacting systems in space. In our experimental setup, even if coalescence events are not individually resolved, they can be detected thanks to the high degree of monodispersivity of bubble size. Indeed, close observation of the images reveals that some bubbles are slightly larger than the majority of the population. These larger bubble are formed via coalescence of two or more primary bubbles as the bubble jet travels away form the injector and are due to the inherent turbulence of the flow. Coalescence events are typically observed more often in the region close to the inlet.

For a cone of bubbles, the Schlichting solution for the mean flow predicts that the linear bubble density in the axis direction; that is, the number of bubbles contained in a section of the jet of thickness $\Delta x$ at a distance $x$, must grow linearly with $x$. This is a direct consequence from the fact that $u \sim 1 / x$, and so for a constant bubble flux $J_{B}=\rho_{x} u$, where $\rho_{x}$ is the number of bubbles per unit length, we have $\rho_{x} \sim x$. Consequently, regardless of the degree of radial spreading of the bubbles, in the absence of coalescence, conservation of the bubble number implies the linear growth of the linear density; this is what is obtained in Fig. 9. The good agreement, within experimental uncertainty, with the preceding prediction indicates that the degree of bubble coalescence is quite small and does not show up significantly in this type of integral characterization. Note also that because the area of the jet section occupied by bubbles grows as $x^{2}$, the average number of bubbles per unit volume decreases as $1 / x$; hence, the 


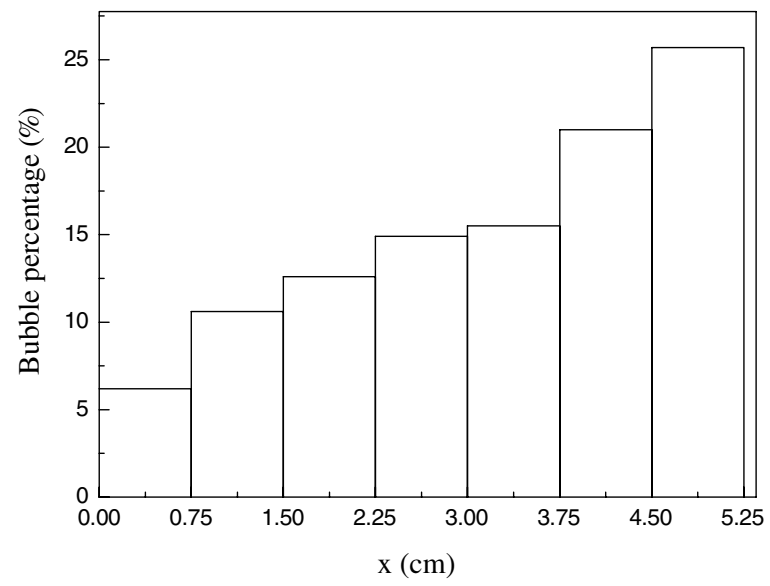

Fig. 9 Percentage of bubbles contained in sections perpendicular to the jet axis, as a function of the distance to the injection point.

typical bubble-bubble distance grows as $x^{1 / 3}$. As a consequence, coalescence events become less and less likely with distance from the injection point. This is to be compared with the case of a jet in laminar flow, in which bubbles would remain aligned in the jet axis. In that case, the bubble separation would decrease as $1 / x$, implying the pileup of bubbles and consequently their massive coalescence. We thus see that in the present experimental setup, the presence of some degree of turbulence plays a crucial role in both spreading the bubbles and drastically diminishing the likelihood of coalescence.

A possible way to characterize the degree of coalescence that is more sensitive than the distribution of bubble density shown in Fig. 10 is to compare measurements of the average bubble size immediately after injection and after $4 \mathrm{~s}$ of microgravity. Figure 10 depicts this comparison as a function of the crossflow liquid velocity at detachment. As expected, the bubble-size diameter $d_{B}$ exhibits a relatively large scattering because the bubble coalescence mechanism is inherently noisy. It is to be remarked that a significant number of coalescence events in the last second occur as a result of the accumulation of bubbles that have reached the end of the cavity. Consequently, the degree of coalescence in a jet formed in a larger cavity is expected to be significantly smaller.

As a general conclusion, we can state that the overall tendency to coalescence is much smaller than in previous studies because the degree of turbulence of the cavity flow is also smaller. Bubbles remain spherical and small compared with their mean separation in the jet, and turbulence is sufficient to keep their spatial distribution relatively sparse, so that bubble-bubble encounters are relatively unlikely. A more detailed study of bubble coalescence should focus on the statistics of bubble encounters as bubbles undergo a

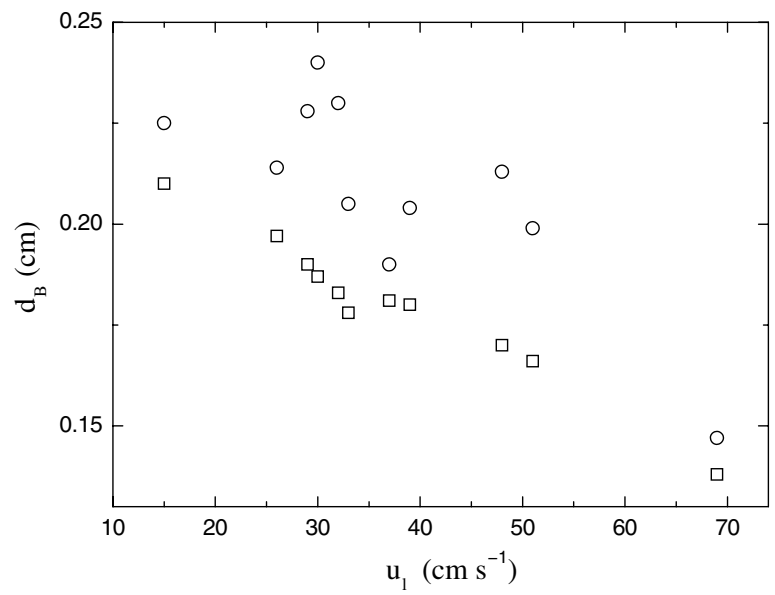

Fig. 10 Average bubble diameter vs crossflow liquid velocity immediately after injection (squares) and $t=4 \mathrm{~s}$ of microgravity (circles); the difference is indicative of the degree of coalescence.

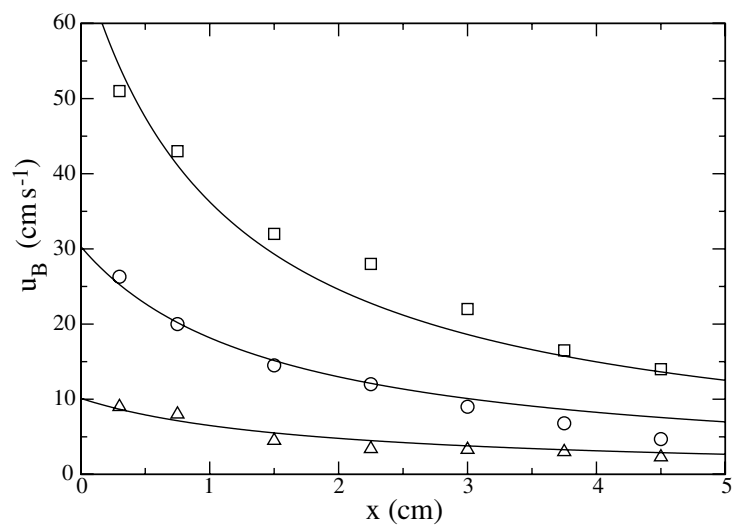

Fig. 11 Bubble velocity at the visual jet centerline vs distance from the injection point: test 3 (squares), tests 1 and 2 (circles), and test 7 (triangles) (see Table 1); nonlinear fits of the Schlichting solution Eq. (21) (solid lines); results of the fits are $x_{0}=1.11,1.50$, and $1.80 \mathrm{~cm}$, respectively.

nonhomogeneous, biased random walk, possibly incorporating hydrodynamic interactions. Note that the statistics of bubble coalescence is entirely dominated by the fluctuating part of the turbulent component of the flow and that not only the stationary statistics of this component is required, but details on the velocity correlations may be crucial because they directly affect the properties of the bubble trajectories. A proper stochastic modeling of the carrying flow is thus called for. This point is of much practical relevance and is deferred to future work.

To complete the characterization of the bubble jet, in Figs. 11 and 12 we plot the bubble velocity at the jet axis and at the jet boundary (the surface of the bubble cone) as a function of horizontal distance to the inlet. Indeed, the measurements of mean bubble velocity at a given point will average out the fluctuating turbulent component and will therefore capture the mean flow. Although the turbulent component is responsible for the bubbles exploring regions relatively far form the jet axis, averaged measurements at a given location are expected to act as passive tracers of the mean flow, which in turn must be close to the Schlichting solution. We find that our direct measurements of the mean flow through bubble velocities are indeed in excellent agreement with the Schlichting solution. Effects due to finite size of the cavity are negligible, whereas a correction due to the finite diameter of the injector tube is obviously required to regularize the divergence of the Schlichting solution at the inlet, $x=0$. This introduces the only fitting parameter in the form of a small shift of origin of $x$ on the order of the capillary diameter [21]. The prefactor $J$ in the solution is not fitted from the measured velocity profile. Instead, we use our measured value according to our

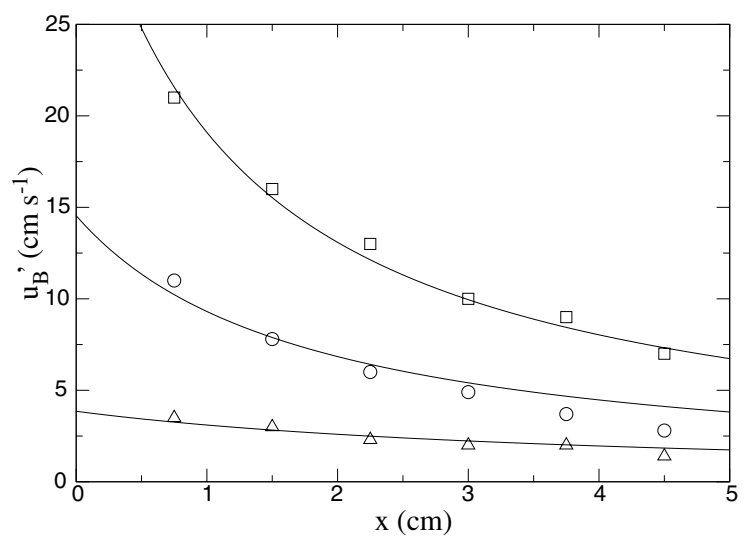

Fig. 12 Bubble velocity at jet boundary vs distance from the injection point along the axis: test 3 (squares), tests 1 and 2 (circles), and test 7 (triangles) (see Table 1); nonlinear fits of the Schlichting solution Eq. (21) (solid lines); results of the fits are $x_{0}=1.18,1.78$, and $4.13 \mathrm{~cm}$, respectively. 


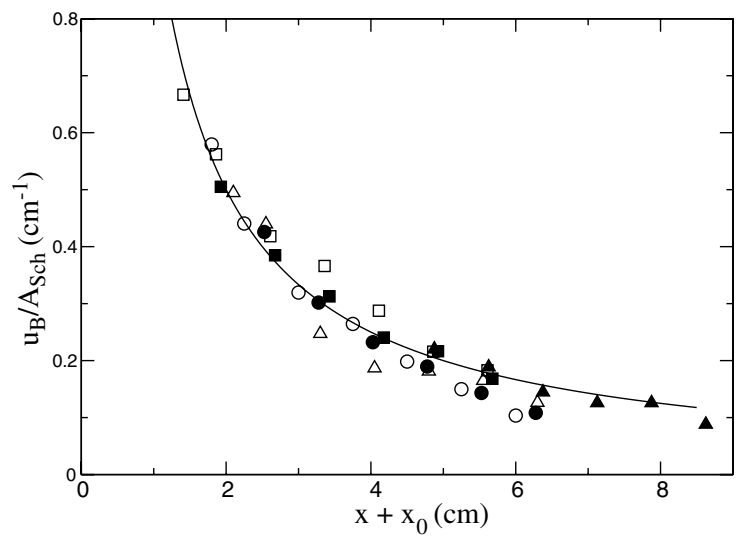

Fig. 13 Collapse of all measurements of averaged local velocities of bubbles when normalized by the prefactor $A_{\text {Sch }}$ defined in Eq. (21); measurements at the jet axis (white points) and at the jet boundary (black points) for different jet angles; Schlichting solution (solid line).

definition of $J$ that combines both gas and liquid injection jets. Although using the two fitting parameters would have certainly improved the fit, the agreement with the theoretical value of $J$ is a direct test of the definition of $J$, which takes into account the dependence of the injected volume fraction in the slug flow.

Note that measuring bubble velocities at the jet boundary for different values of the bubble jet angle allows us to probe the jet velocity at different radial distances from the axis, because the structure of the underlying liquid jet solution is independent of $J$, except for an overall velocity scale. Accordingly, we should be able to find a single collapse of the six curves of Figs. $\underline{11}$ and $\underline{12}$ by inserting

$$
\zeta=\frac{1}{4 c} \sqrt{\frac{3}{\pi}} \tan \frac{\phi(J)}{2}
$$

into

$$
u=\frac{3}{8 \pi c} \sqrt{\frac{J}{\rho_{l}}} \frac{1}{\left(1+\zeta^{2} / 4\right)^{2}} \frac{1}{x+x_{0}} \equiv A_{\mathrm{Sch}}(J, \phi(J)) \frac{1}{x+x_{0}}
$$

where $x_{0}$ is fitted from the experimental data, and where $\phi(J)$ is taken from the data of Fig. 8. In Fig. 13 we show an excellent collapse of the curves to the Schlichting solution, with the effective prefactor given by $J=\rho_{l} Q_{l}\left(Q_{l}+Q_{g}\right) / A$. Note that we find a collapse even for the measurements at the jet boundary, for which we are using a single value of $\zeta$ for all points with the same $J$, disregarding the fact that the jet boundary is only roughly defined, and the jet angle $\phi(J)$ is measured as an average value along the jet, over points for which $\zeta$ is actually scattered. We thus conclude that as far as the mean flow is concerned, the only effect of the gas injection is to modify the effective momentum rate of the jet, whereas averaged local values of bubble velocities trace the mean flow of the Schlichting solution to a very good approximation.

\section{Conclusions}

In the present study, we theoretically and experimentally investigated a method of bubble formation and spreading in microgravity environments based on a composite gas-liquid injector. The basic idea of the method is to create a slug flow at a $\mathrm{T}$ junction where capillary forces are dominant over inertial forces. Bubble detachment is achieved when the drag force exerted by the crossflow balances the surface-tension force. In this way, the method works efficiently at small nominal values of the Weber number, as opposed to previous studies, whereas the geometrical constraints force the effective Weber number $W e^{*}$ that controls bubble detachment to become very large. The departure between the nominal and the effective Weber number is at the root of the efficiency regarding the production of bubble sizes on the order of the capillary tube and also in producing them with very small size dispersion. Our theoretical analysis explains both observations in a quantitative framework. The two unknown parameters of the analysis are then determined by fits of the experimental data obtained in both normal gravity and microgravity. The method of bubble formation is shown to be insensitive to the gravity conditions, and the bubble size exhibits only a weak linear decrease of bubble size with $\left[W e^{0}\right]^{1 / 2}$.

In the second part of the paper, we discussed the characteristic features of the turbulent bubble jet created by this method. The result of injecting the slug flow into a quiescent cavity is to form a turbulent jet of virtually monodisperse spherical bubbles. The inertia injected by the liquid part of the slug flow generates a turbulent liquid jet in the cavity that acts as a carrier flow of the bubbles. Measurements of averaged local velocities of bubbles show that these passively trace the mean flow of the carrying liquid jet. That is, the only effect of bubbles in the mean flow is to set the effective momentum injection jet, which depends on the gas volume fraction in the slug flow. The presence of turbulence in the jet, however, is crucial to achieve efficient spreading of the bubble spatial distribution and to therefore minimize the degree of bubble coalescence. The envelope of the bubble suspension takes a conical shape, with an opening angle that increases with the Reynolds number and saturates to maximum value around $10 \mathrm{deg}$ at $R e \simeq 800$. In all our experiments, coalescence is shown to be much reduced with respect to previous studies. A more detailed study of the statistics of bubble coalescence within an accurate stochastic modelization of the turbulent component of the flow, possibly including hydrodynamic interactions and velocity correlations, is an interesting follow-up of the present study, particularly for its relevance in technological applications.

\section{Acknowledgments}

We gratefully acknowledge the support from the DLR, German Aerospace Center for the funding of the drop-tower flights and from the Spanish Ministry of Science and Technology through the Space National Program (projects ESP2002-10776-E, ESP2004-0074, and ESP2005-25290-E). X. Ruiz and J. Casademunt also acknowledge additional support from project FIS2006-03525 and L. RamírezPiscina acknowledges support from project FIS2006-11452-C0302, both from Ministerio de Ciencia y Tecnología.

\section{References}

[1] Rabiger, N., and Vogelpohl, A., Encyclopedia of Fluid Mechanics Vol. 3, Gulf Publishing, Houston, TX, 1986, pp. 59-88.

[2] Kumar, R., and Kuloor, N. R., "The Formation of Bubbles and Drops," Advances in Chemical Engineering, Vol. 8, 1970, pp. 255-368. doi:10.1016/S0065-2377(08)60186-6

[3] Kim, I., Kamotani, Y., and Ostrach, S., "Modeling Bubble and Drop Formation in Flowing Liquids in Microgravity," AIChE Journal Vol. 40, No. 1, 1994, pp. 19-28. doi:10.1002/aic.690400105

[4] Pamperin, O., and Rath, H.-J., "Influence of Buoyancy on Bubble Formation at Submerged Orifices," Chemical Engineering Science, Vol. 50, No. 19, 1995, pp. 3009-3024. doi:10.1016/0009-2509(95)00140-Z

[5] Nahra, H. K., and Kamotani, Y., "Prediction of Bubble Diameter at Detachment from a Wall Orifice in Liquid Cross-Flow Under Reduced and Normal Gravity Conditions," Chemical Engineering Science, Vol. 58, No. 1, 2003, pp. 55-69. doi:10.1016/S0009-2509(02)00516-X

[6] Buyevich, Y. A., and Webbon, B. W., "Bubble Formation at a Submerged Orifice in Reduced Gravity," Chemical Engineering Science, Vol. 51, No. 21, 1996, pp. 4843-4857. doi:10.1016/0009-2509(96)00323-5

[7] Tsuge, H., Terasaka, K., Koshida, W., and Matsue. H., "Bubble Formation at Submerged Nozzles for Small Gas Flow Rate Under Low Gravity," Chemical Engineering Science, Vol. 52, No. 20, 1997, pp. 3415-3420. doi:10.1016/S0009-2509(97)00159-0

[8] Tsuge, H., Tanaka, Y., Terasaka, K., and Matsue. H., "Bubble Formation in Flowing Liquid Under Reduced Gravity," Chemical Engineering Science, Vol. 52, Nos. 21-22, 1997, pp. 3671-3676. 
doi:10.1016/S0009-2509(97)00213-3

[9] Bhunia, A., Pais, S. C., Kamotani, Y., and Kim, I.-H., "Bubble Formation in a Coflow Configuration in Normal and Reduced Gravity," AIChE Journal, Vol. 44, No. 7, 1998, pp. 1499-1509. doi:10.1002/aic.690440704

[10] Pais, S. C., Kamotani, Y., Bhunia, A., and Ostrach, S., "Bubble Generation in a Flowing Liquid Medium and Resulting Two-Phase Flow in Microgravity," Proceedings of the Fourth Microgravity Fluid Physics and Transport Phenomena Conference, NASA, CP-19992085261999, pp. 70-75; also available online at http://gltrs.grc.nasa.gov/reports/1999/CP-1999-208526.pdf.

[11] Nahra, H. K., and Kamotani, Y., "Bubble Formation from Wall Orifice in Liquid Cross-Flow Under Low Gravity," Chemical Engineering Science, Vol. 55, No. 20, 2000, pp. 4653-4665. doi:10.1016/S0009-2509(00)00102-0

[12] Carrera, J., Parthasarathy, R. N., and Gollahalli, S. R., "Submerged Gas Injection from a Tube in Microgravity," Sixth Microgravity Fluid Physics and Transport Phenomena Conference, Vol. 2, NASA, TM2002-211211, 2002, pp. 571-581; also available online at http:// gltrs.grc.nasa.gov/reports/2002/TM-2002-211211.pdf.

[13] Carrera, J., Parthasarathy, R. N., and Gollahalli, S. R., "Bubble Formation from a Free-Standing Tube in Microgravity," Chemical Engineering Science, Vol. 61, No. 21, 2006, pp. 7007-7018. doi:10.1016/j.ces.2006.07.021

[14] Herman, C., Iacona, E., Földes, I. B., Suner, G., and Milburn, C., "Experimental Visualization of Bubble Formation from an Orifice in Microgravity in the Presence of Electric Fields," Experiments in Fluids, Vol. 32, No. 3, 2002, pp. 396-412. doi: $10.1007 / \mathrm{s} 003480100366$

[15] Herman, C., and Iacona, E., "Modeling Of Bubble Detachment in Reduced Gravity Under the Influence of Electric Fields and
Experimental Verification," Heat and Mass Transfer, Vol. 40, No. 12, 2004, pp. 943-957. doi:10.1007/s00231-003-0488-y

[16] Di Marco, P., Grassi, W., Memoli, G., Takamasa, T., Tomiyama, A., and Hosokawa, S., "Influence of Electric Field on Single Gas-Bubble Growth and Detachment in Microgravity," International Journal of Multiphase Flow, Vol. 29, No. 4, 2003, pp. 559-578. doi:10.1016/S0301-9322(03)00030-2

[17] Moore, D. W., "The Boundary Layer on a Spherical Gas Bubble," Journal of Fluid Mechanics, Vol. 16, No. 2, 1963, pp. 161-176. doi:10.1017/S0022112063000665

[18] Magnaudet, J., and Eames, I., "The Motion of High-Reynolds-Number Bubbles in Inhomogeneous Flows," Annual Review of Fluid Mechanics, Vol. 32, 2000, pp. 659-708. doi:10.1146/annurev.fluid.32.1.659

[19] Schlichting, H., Boundary-Layer Theory, 7th ed., McGraw-Hill, New York, 1979.

[20] Versteeg, H. K., and Malalasekera, W., An Introduction to Computational Fluid Dynamics: The Finite Volume Method, Pearson Prentice-Hall, Lebanon, IN, 1995.

[21] Abramovich, G. N., and Shindel, L., The Theory of Turbulent Jets, MIT Press, Cambridge, MA, 1963

[22] Martínez-Mercado, J., Palacios-Morales, C. A., and Roberto Zenit, "Measurement of Pseudoturbulence Intensity in Mondispersed Bubbly Liquids for $10<R e<500$," Physics of Fluids, Vol. 10, 2007, Paper 103302. doi: $10.1063 / 1.2772256$ 